\title{
APRENDER EN CONTEXTOS
}

\section{desfavorecidos}

DOI

Equipo docente del Ceip Antonio Machado de Mérida*

cp.antoniomachado@edu.gobex.es

\section{El CENTRO Y SU CONTEXTO}

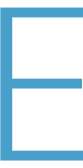

I CEIP Antonio Machado se encuentra situado entre dos barriadas donde se realojó una antigua zona marginal de la ciudad de Mérida.

Las características socioeconómicas del barrio, mayoritariamente de etnia gitana, se encuadran dentro de los índices de pobreza y exclusión social. Junto a unos niveles educativos muy bajos e importantes tasas de analfabetismo, cuando menos funcional, hay un elevadísimo porcentaje de paro, de economía sumergida y de subsistencia. Nos encontramos con un alto índice de delincuencia y tráfico de drogas, muchas familias desestructuradas, traslados temporales de domicilio, maternidad a edades muy tempranas... Nula participación ciudadana, escaso interés por la formación y la educación como herramienta para la mejora de la calidad de vida personal y profesional.

El alumnado es mayoritariamente de etnia gitana (96\%).

Partíamos de niveles de escolarización temprana muy bajos; índices de absentismo escolar y abandono temprano de la escolarización, alarmantes. En general, el alumnado manifestaba escasa motivación hacia los aprendizajes escolares, problemas individuales de conducta, habilidades sociales poco desarrolladas e importantes dificultades en los aprendizajes instrumentales.

La participación de las familias en el centro se reducía a situaciones concretas, destacando su escasa implicación en la formación de sus hijos, y relativo rechazo a la institución escolar, desórdenes alimenticios y horarios inadecuados de sueño, reflejo de cierta dejación de responsabilidades paternales.

Todo ello hace del centro educativo un gueto social, cultural, laboral, económico, académico y educativo.

\footnotetext{
* Marina Fernández Casero, M. a del Mar Rodríguez Santizo, M. ${ }^{a}$ Elena Pérez González, Rosario Aparicio Parra, Paula García Álvarez, Olga Durán Luis, Verónica del Castillo Cor-

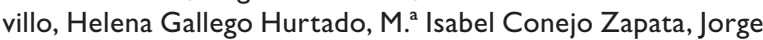
Cortés Constantino, Dolores Barroso Martínez, Juan Pablo Correa Suárez, Begoña García Gudiño, María Jesús Sánchez Rivera, Joaquín Ayuso Sánchez, Mar Medrano Millán, Carmen Martín Chacón y Pedro José Lozano Goñi.
}

- Nuestro centro, gueto social, cultural y económico pone en marcha actuaciones basadas en experiencias de éxito para romper con el círculo de la desigualdad. Proporcionando una educación de calidad, sin dar cabida a la queja y desde planteamientos del proyecto "Comunidades de Aprendizaje", hemos logrado reducir el absentismo, mejorar los aprendizajes, la convivencia y la participación de familias. Tenemos muchas razones para creer en la transformación.

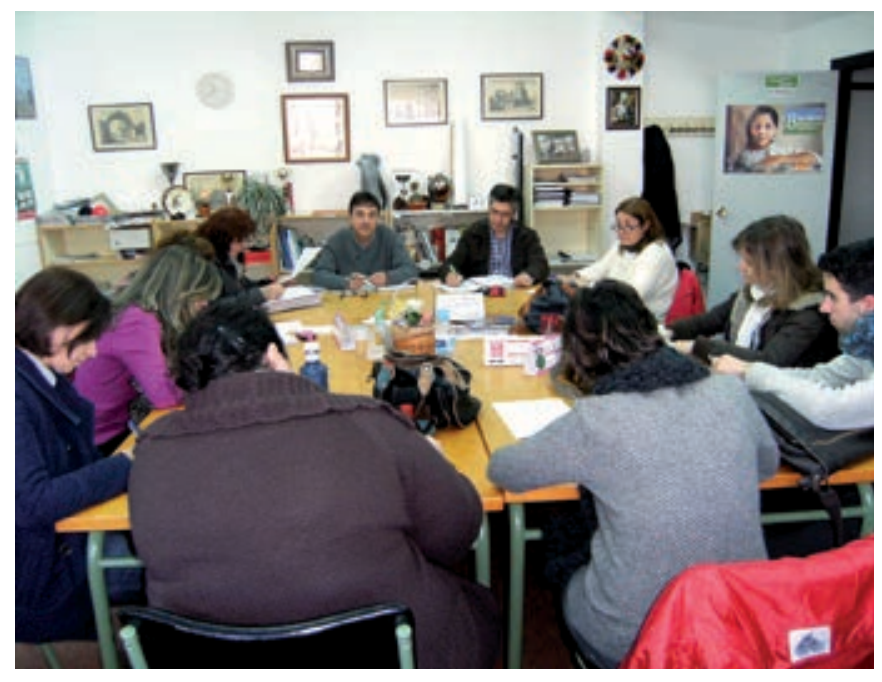

Equipo docente CEIP Antonio Machado de Merida. 
Figura I. Campos de actuación

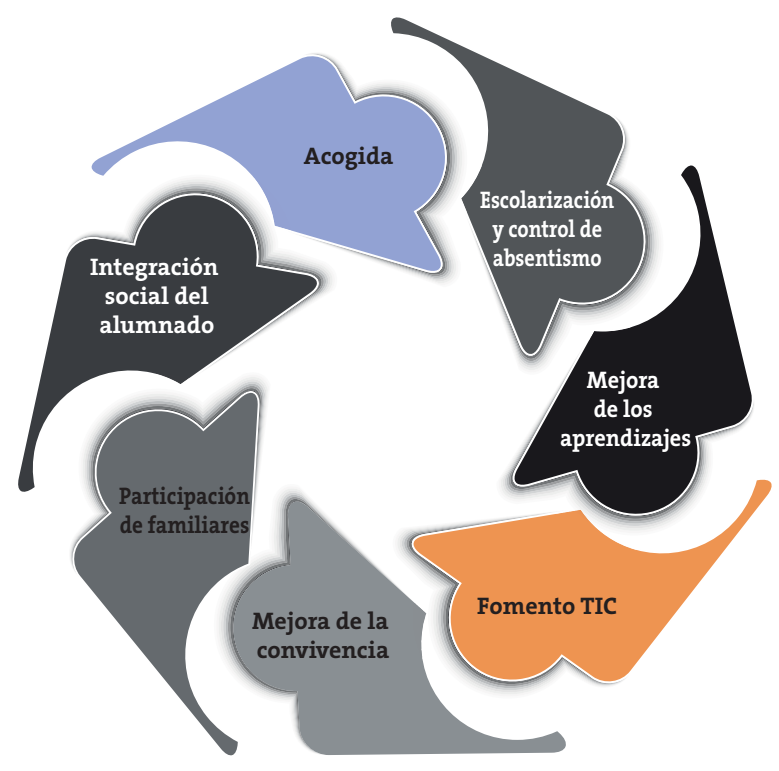

ANÁlISIS DE PARTIDA

Partimos de las siguientes premisas:

- El fracaso escolar del alumnado no debe atribuirse exclusivamente al alumnado, también están fracasando la educación, la escuela que lo escolariza, la familia y la sociedad en general.

- El fracaso escolar afecta a todas las categorías sociales, aunque el alumnado procedente de entornos desfavorecidos está más expuesto a él.

- Las escuelas públicas situadas en zonas socialmente desfavorecidas tienen más dificultades para romper el círculo de la desigualdad social.

- Sólo es posible romper el círculo de la desigualdad social proporcionando una educación de máxima calidad.

Esta realidad compleja y difícil llevaba al profesorado habitualmente a la queja y la desesperanza cuando, por el contrario, exige actuaciones innovadoras para lograr paulatinamente la transformación del centro y entorno.

\section{Nuevos objetivos, Nuevos Retos}

En este entorno, nuestros objetivos son:

- Lograr el mayor éxito educativo para todos (pedagogía de máximos).

- Reducir el absentismo y abandono escolar.

- Mejorar la convivencia entre todos los miembros de la comunidad.

- Abrir el centro a la participación de las familias y del entorno.

- Incluir a nuestros alumnos en el centro y en otros entornos más amplios.

- Pasar de la cultura de la queja a la cultura de la transformación.
Queríamos que los niños y las niñas viniesen todos los días a clase, queríamos disminuir el absentismo e incorporarles cuanto antes al colegio (escolarización a los tres años), sabedores de que el aumento de las interacciones desde edades tempranas es determinante en el aprendizaje.

Sabíamos que si los alumnos venían todos los días a clase, y en clase dejábamos de adaptarnos a sus niveles bajos, mejorarían los resultados. Sabíamos que si sustituíamos el currículum de la felicidad por un currículum instrumental, y organizábamos el aula y el centro para promover todas las interacciones posibles alumnado-alumnado, alumnado-profesorado, alumnado-voluntariado, se mejoraría el aprendizaje y la convivencia. También sabíamos que, con la participación de las familias en el aprendizaje y en las decisiones del centro, la mejora en el aprendizaje, en la convivencia y en la transformación del barrio se incrementaría.

\section{¿Qué HACEMos?}

Lo que hacemos son prácticas de éxito, avaladas por la comunidad científica internacional capaces de superar las desigualdades con que nuestro alumnado llega al centro. Todas estas prácticas, y otras que nos son propias, se desarrollan desde planteamientos del "aprendizaje dialógico" y son:

- Actuaciones en relación con la acogida de alumnado, de los familiares y del profesorado de nueva incorporación al centro.

- Actuaciones para el fomento de la escolarización y para el control de absentismo:

- Campaña de sensibilización para la escolarización en Educación Infantil.

- Comisión, protocolo y plan de absentismo del centro.

- Proyecto "Súbete al carro", para sensibilizar sobre la importancia de la educación y la continuidad en ESO.

- Actuaciones en relación con el fomento de las TIC: blog del centro, pizarra digital, aula de informática, tabletas digitales.

- Actuaciones en relación con la mejora de los aprendizajes:

- Grupos Interactivos.

- Tertulias literarias dialógicas.

- Programa de desarrollo del lenguaje oral.

- Huerto y semillero dialógico.

- Ludoteca.

- Incremento del tiempo escolar: programa de apoyo socioeducativo (REMA y PROMOCIONA) y actividades formativas complementarias.

- Actuaciones en relación con la mejora de la convivencia: 
- Comisión, protocolo y programa de convivencia.

- Programa de competencia social.

- MUS-E.

- Asambleas de aula, ciclo y/o centro.

- Actuaciones en relación con la participación de familiares:

- Asamblea de familiares.

- Formación de familiares.

- Comisiones: absentismo, convivencia y celebración del $\mathrm{XXV}$ Aniversario del centro.

- Actuaciones en relación con la integración social del alumnado:

- Días singulares: Día Escolar de la Bicicleta, Día Mundial de la Poesía, Día Internacional del Pueblo Gitano.

- Proyectos institucionales: "la escuela adopta un monumento", "bosque y vida", "la llegada del otro...".

- Encuentros y visitas: escuela taller, encuentro con Marruecos, intercambios con otros centros (tertulias literarias y grupos interactivos), Visita alumnado Facultad de Educación de Segovia...

- Actividades extraescolares y celebraciones pedagógicas.

- Taller de competencias sociales con Manuel Segura.

\section{LOGROS}

- Mayor éxito educativo de todos nuestros alumnos

La evaluación de técnicas instrumentales en los últimos tres cursos revela una mejora en los resultados en todos los niveles educativos $y$, cada año, se va aumentando el número de cursos que, de manera global, se acercan al nivel que corresponde a su curso.

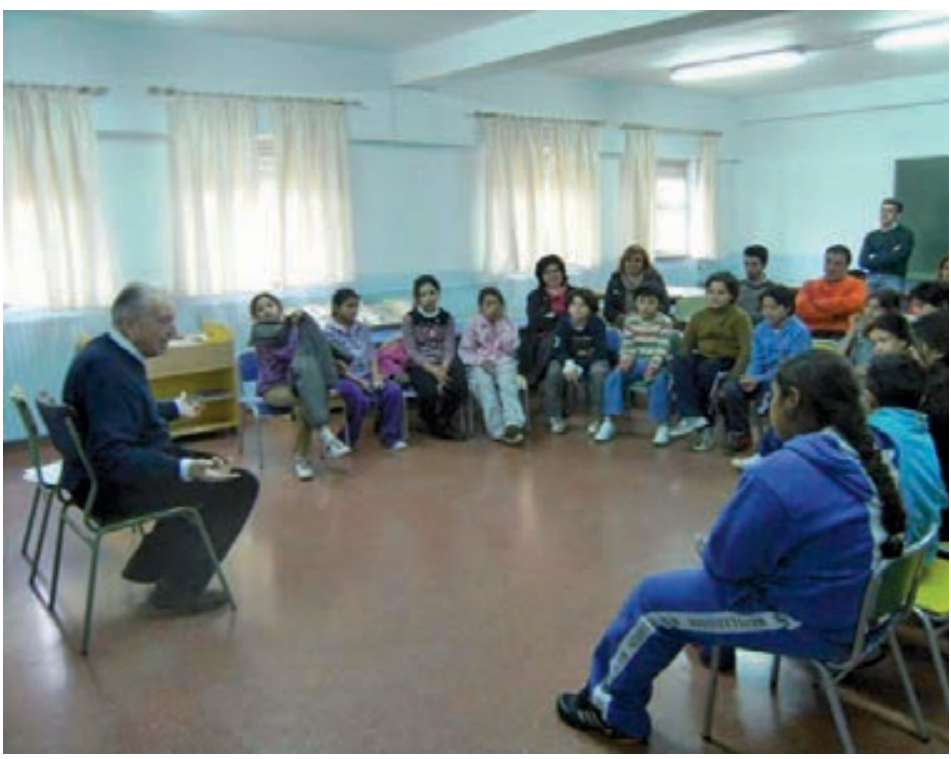

\section{actividades de aula}

\section{SÚBETE AL CARRO: I. ' SESIÓN}

- Participantes: alumnos $6^{\circ}$ curso, madres y padres, tutora, apoyos profesorado, EOEP, Programa de Desarrollo Gitano, voluntarias, grupo "Súbete al carro", Delegación de Educación, Inspección, FAGEX.

- Desarrollo sesión:

- Valoración positiva del grupo de $6^{\circ}$, alumnas y alumnos y posibilidades de éxito en Enseñanza Secundaria

- Visionado del audiovisual "A la escuela voy". Dada su corta duración, se puede volver a reproducir o destacar alguna conversación.

- Hablamos sobre lo visto, comentando aquello que nos ha llamado la atención, gustado, frases, contexto, semejanzas...

- Preguntamos o introducimos temas:

- Qué pretende el audiovisual

- Qué personaje y frase te ha resultado más significativa

- Encuentras parecidos o diferencias con tu entorno

- Valoremos lo que nos dicen algunos personajes: Jesús Amador, Tío Moreno, Tía Tigilí (ellos no pudieron estudiar)

- Consideramos importante la escuela para el futuro.

- Referentes invitados explican su experiencia vital.

- Propuesta de actividad: "Los estudios de nuestros mayores".

Proponemos la realización de un cuestionario a nuestros familiares cercanos, madre, padre, abuelos, hermanos mayores, tíos y primos o incluso a algún vecino que conozcamos.

Se trata de recoger las experiencias de nuestra familia con la escuela y la educación. Para ello utilizaremos un sencillo cuestionario con preguntas, en las que escribiremos sobre aquello que nos ha contado un familiar acerca de la pregunta realizada. Al final de la respuesta anotaremos la persona que ha respondido a la pregunta.

Se ha logrado:

- Acelerar el proceso de aprendizaje en las áreas instrumentales. El alumnado está todo el tiempo aprendiendo y aprende más.

- Desarrollar en el alumnado responsabilidades individuales y colectivas, fomentado la solidaridad, el respeto y la cooperación.

- Aumentar el nivel de expectativas que el alumnado tiene sobre sí mismo, así como las expectativas que los adultos tienen de ellos.

\section{- Reducción Absentismo}

El desarrollo sistemático del plan de absentismo ha propiciado la mejora la asistencia regular. Los datos de absentismo globales del centro en el curso 2012-2013 se sitúan en el $16,49 \%$ (14,82\% en Infantil y $17,74 \%$ en Primaria) No hay que olvidar que se habían alcanzado porcentajes cercanos al $40 \%$. Aunque son cifras que aún se alejan de la normalidad constatamos que: 


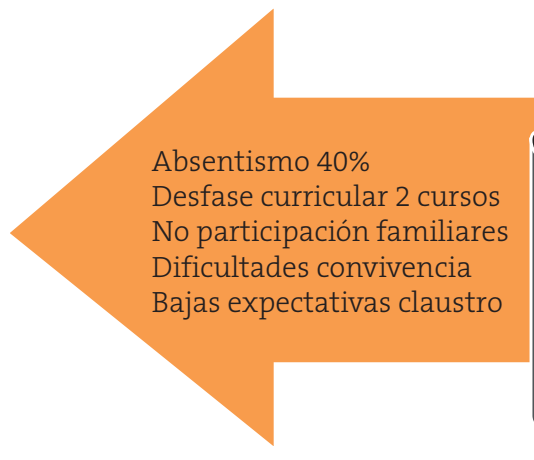

- Desciende el número de alumnos con absentismo crónico y absoluto.

- Cada vez un número mayor de alumnado demanda a sus familias venir al centro diariamente, siendo en muchas ocasiones los menores los que despiertan a los padres para poder asistir al centro. Crece el número de alumnos con asistencia regular.

- Existen grupos-clase con una asistencia prácticamente normalizada.

- Las familias son más conscientes de la importancia del proceso educativo de sus hijos e hijas y por tanto de la asistencia regular al centro, que se pone de manifiesto en la disminución de absentismo, en su disposición para justificar ante el centro aquellas faltas causadas por enfermedad, en comunicar al centro los traslados a otras ciudades por motivos laborales o familiares, etcétera.

- Se ha normalizado el horario escolar y, aunque las puertas del centro no se cierran, se va avanzando también en que el alumnado acuda puntualmente al centro.

\section{- Mejora de la convivencia entre todos los miembros de la comunidad}

Se han reducido los conflictos entre todos los miembros de la comunidad educativa $y$, cuando éstos se producen, se solucionan desde el diálogo. Se busca crear un clima de convivencia asertivo que genere un ambiente que facilite los aprendizajes y las relaciones interpersonales.

\section{- Mejora del nivel de participación e implica- ción de las familias}

A través de la Asamblea de Familiares y de muchos de los encuentros informales con las familias se ha aumentado su participación. Estos encuentros han hecho posible que familias, profesorado y personal laboral hagamos explícitas las metas que perseguimos.

Cada vez más, la participación de familiares responde a modelos de participación en el aprendizaje (como voluntarios en grupos interactivos, tertulias literarias dialógicas, lectura dialógica) y en la toma de decisiones (como miembros de las comisiones de convivencia, de absentismo y del XXV Aniversario del centro).

\section{- Mayor integración del alumnado en espacios educativos de la ciudad}

Nuestro alumnado ha adquirido habilidades necesarias para adecuar su comportamiento a salidas y celebraciones pedagógicas, para organizar eventos y participar en programas institucionales. Progresivamente, ha sido más activo en estos nuevos espacios de relación y ello ha contribuido a que la ciudad empiece a tener una visión más positiva de nuestro centro.

\section{- Cambio de actitud en el profesorado}

A pesar de las dificultades, los espacios de encuentro del profesorado (Claustro, CCP) han dejado de estar invadidos por la queja, y actualmente son espacios de reflexión y trabajo conjunto para afrontar las dificultades.

Se da una implicación personal y profesional del profesorado imprescindible para alcanzar los objetivos y metas planteadas.

\section{Para saber más}

- Aubert, Adriana; Flecha, A; García, C; Flecha, R, y RACIONERO, S. (2008). Aprendizaje dialógico en la sociedad de la información. Barcelona: Hipatia.

- Freire, P. (1997). A la sombra de este árbol. Barcelona: El Roure.

- Vigotsky, L. (1979). El desarrollo de los procesos psicológicos superiores. Barcelona: Crítica.

\section{hemos hablado de:}

\section{Aprender; dialogar; éxito educativo;} transformar.

Este artículo fue solicitado por PADRES Y MAESTROS en julio de 2013, revisado y aceptado en diciembre de 2013 para su publicación. 